\title{
Perceptions of Training Pathways from Recent Cardiothoracic Surgery Graduates
}

\author{
Alison F. Ward, MD,${ }^{1}$ Neel K. Ranganath, MD, ${ }^{2}$ Stacey Chen, $M D,{ }^{2}$ Tyler Wallen, DO, ${ }^{3}$ Amie J. Kent, MD, ${ }^{2}$ \\ Deane E. Smith III, MD, ${ }^{2}$ Zachary N. Kon $\mathrm{MD}^{4}$ \\ ${ }^{1}$ Division of Cardiothoracic Surgery, Augusta University Health, Augusta, GA, USA; \\ ${ }^{2}$ Department of Cardiothoracic Surgery, NYU Langone Health, New York, NY, USA; \\ ${ }^{3}$ Division of Cardiothoracic Surgery, University of Florida, Gainesville, FL, USA; \\ ${ }^{4}$ Department of Cardiothoracic Surgery, Northwell Health, Manhasset, NY, USA
}

\section{ABSTRACT}

Background: There are three cardiothoracic surgery (CTS) training pathways-general surgery residency followed by a CTS residency of 2-3 years (traditional), 4 years of general surgery and 3 years of CTS residency $(4+3)$, and an integrated 6-year pathway (I-6). The goal of this study was to survey early career cardiothoracic surgeons regarding their training experiences.

Methods: An email-based survey was sent to cardiothoracic surgeons, who graduated between 2012-2017. Data on training pathway specific variables and overall satisfaction were collected. The primary endpoints were career preparation and satisfaction, scored on a scale from 1-100, 100 being the most positive.

Results: Four hundred seventy-seven emails were sent, with a response rate of $95 / 477(20 \%)$. Seventy-six of the respondents $(80 \%)$ were male; the mean age was 39 . Seventyseven $(81.0 \%)$ completed a traditional training pathway, 7 (7.4\%) completed a $4+3$ pathway, and $11(11.6 \%)$ completed an I-6 pathway. Participants felt prepared for practice with a mean response of 79.8 (range 31-100); mean career satisfaction was 87.6. When asked which pathway respondents would choose in the current era, $52(54.7 \%)$ would choose a traditional pathway, $17(17.9 \%)$ a $4+3$ pathway, and 19 (20.0\%) an I-6 program; 7 (7.4\%) did not respond. Twenty of $72(27.8 \%)$ traditional pathway trained and 18/18(100\%) integrated pathway trained surgeons would choose an integrated pathway.

Conclusions: This is the first survey addressing perceptions of training from early career cardiothoracic surgeons across all training pathways. Data from this study provides insights to better understand how to improve CTS training for the next generation of surgeons.

Received Fanuary 31, 2021; accepted February 16, 2021

Correspondence: Alison F. Ward, MD, Augusta University, 1120 15th Street, Augusta, GA, 30912, Telephone 706-721-3266, Fax 706-721-7508 (e-mail: ALLWARD@augusta.edu).

\section{INTRODUCTION}

Cardiothoracic surgery (CTS) training has evolved significantly since the first thoracic surgery residency was created in 1928 by John Alexander at the University of Michigan [Alexander 1936]. Historically, a single pathway to becoming a board-certified cardiothoracic surgeon existed. This involved completion of a general surgery residency, American Board of Surgery certification, followed by completion of a 2 to 3-year cardiothoracic surgery residency and American Board of Thoracic Surgery (ABTS) certification. Recognizing the expanding complexity of the field, many programs lengthened their residency from 2 to 3 years in the 1980s to meet the needs of the changing specialty and work hour restrictions. Subsequently, with changes in the scope of CTS practice, multidisciplinary approaches to cardiothoracic disease, challenges with recruiting strong candidates, and a shift toward integrated training pathways in other competitive subspecialties, new CTS training pathways were developed. The ABTS with the Thoracic Surgery Residency Review Committee (RRC) sought to restructure the CTS curriculum; this new integrated pathway was focused into a 6-year format, also known as the I-6 pathway [Lebastchi 2014]. In 2003, the ABTS made certification by the American Board of Surgery optional, therefore allowing for the creation of this alternate training pathway [Crawford 2005]. The first I-6 residency program in the nation was formed at Stanford University and was approved by the Thoracic Surgery RRC in 2007 [Zhu 2019]. The first four programs approved to accept an incoming class of I-6 residents were Stanford University, University of Pennsylvania, University of Maryland, and Medical University of South Carolina [Ikonomidis 2014].

Currently, there are three distinct CTS training pathways - general surgery residency followed by CTS residency of 2-3 years (traditional), 4 years of general surgery and 3 years of CTS residency $(4+3)$ at the same institution, and an integrated 6-year pathway (I-6). These are referred to as independent programs, joint thoracic/general surgery track, and integrated pathway, respectively, by the Thoracic Surgery Directors Association (TSDA). There also is an option to complete an integrated vascular surgery residency followed by CTS residency of 2 to 3 years but this is an uncommon pathway. There currently are 74 traditional programs, 13 $4+3$ tracks, and 30 I-6 programs [Thoracic Surgery Directors 


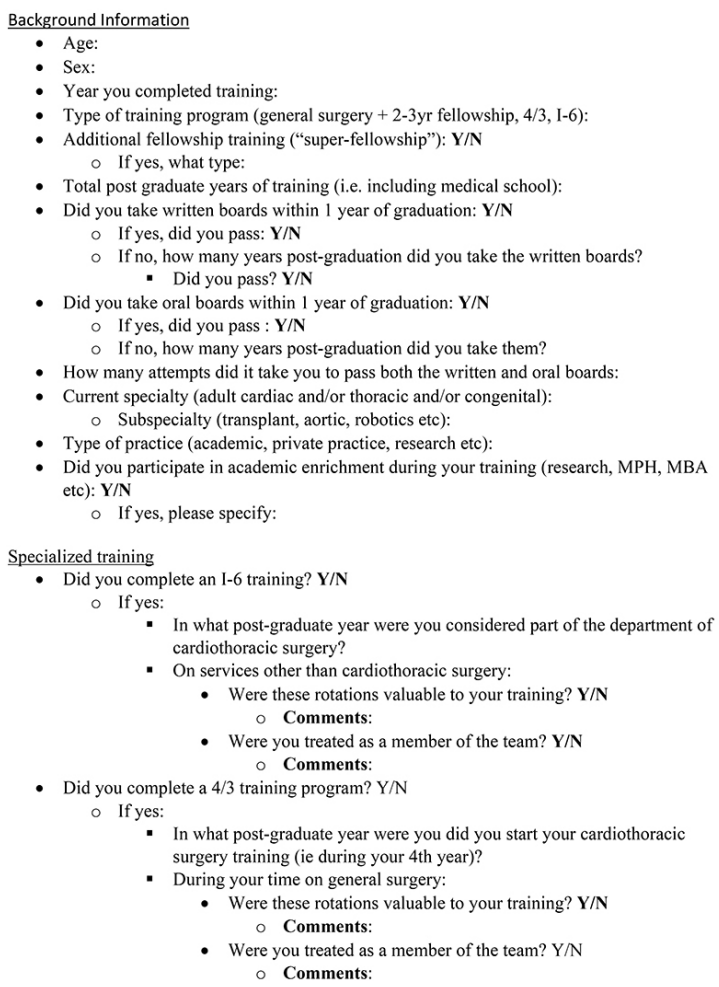

- Did you complete general surgery residency followed by 2-3 years or fellowship? Y/N - If yes, did you feel that residency adequately prepared you for fellowship? Y/N For all Training Pathways

- Did you have formal echocardiography training? $\mathbf{Y} / \mathrm{N}$

- Did you have formal interventional cardiology training? $\mathrm{Y} / \mathrm{N}$

- Did you have formal endovascular training? $\mathbf{Y} / \mathbf{N}$

- If you had formal training in interventional and/or endovascular procedures (ie TAVR, cath, TEVAR etc) do you now perform those procedures as part of your practice? $\mathbf{Y} / \mathbf{N}$

- Did you have training in minimally invasive surgery? $\mathbf{Y} / \mathbf{N}$

0 Do you perform minimally invasive surgery as part of your practice? $\mathrm{Y} / \mathrm{N}$

- Did you have training in robotics? $\mathbf{Y} / \mathbf{N}$

Do you perform robotic surgery as part of your practice? $\mathbf{Y} / \mathbf{N}$

- Did you have training in heart or lung transplant? $\mathbf{Y} / \mathbf{N}$

- Do you perform transplant surgery as part of your practice? Y/N

- Did you have training in mechanical circulatory support? $\mathbf{Y} / \mathbf{N}$ - Do you insert VADs/place patients on ECMO as part of your practice? Y/N

- Did you have formal interventional pulmonology training? $\mathbf{Y} / \mathbf{N}$

- Do you perform such procedures as part of your practice? $\mathrm{Y} / \mathrm{N}$

- Did you have dedicated research time? Y/N

Post-Graduation

- Do you feel your training adequately prepared you as a new attending? Scale 1-10 (1= not prepared at all, $10=$ fully prepared to practice independently)

- Do you feel your training adequately prepared you to sit for the boards during your $1^{\text {st }}$ year in practice? $\mathbf{Y} / \mathbf{N}$

N/A if haven't taken the boards yet or took them after the $1^{\text {st }}$ year

- What were the 3 main deficiencies in your training program? $\circ 1$. ○ 2.

f you had to apply to fellowship now, which training model would you choose? (cenera surgery $+2-3 y$ y fellowship, $4 / 3$, i6) surgery + 2-3yr fellowship, 4/3, i6)

model than the one you completed, why? Would you recommend your training pathway to current medical students? $\mathbf{Y} / \mathbf{N}$ $\circ$ Why or why not?

- Are you satisfied with your career choice? Scale 1-10 (1- not satisfied at all, 10completely satisfied)
Figure 1. Survey

Association 2012]. Each pathway possesses unique advantages and disadvantages, and there is no consensus on which pathway best trains our future cardiothoracic surgeons. There have been multiple survey studies aimed at assessing these training pathways, but their scope often has been limited to current trainees and educators [Lebastchi 2014; Lebastchi 2014; Lee 2003; Nguyen 2015; Sarkaria 2011; Stephens 2015; Stephens 2016; Tchantchaleishvili 2015], lacking real-world fidelity. This limited viewpoint is from others looking in rather than those currently in practice. The goal of this study was to survey early career cardiothoracic surgeons who graduated from any of the three available CTS training pathways within the last 5 years to collect both objective and subjective data in regard to their training experiences.

\section{MATERIALS AND METHODS}

An anonymous online survey of 31-46 questions (depending on the participant's responses) was developed. This study was approved as Institutional Review Board (IRB) exempt and all participants consented prior to the start the survey. Questions addressed in the survey included those about demographics, training pathways, subspecialty training, ABTS

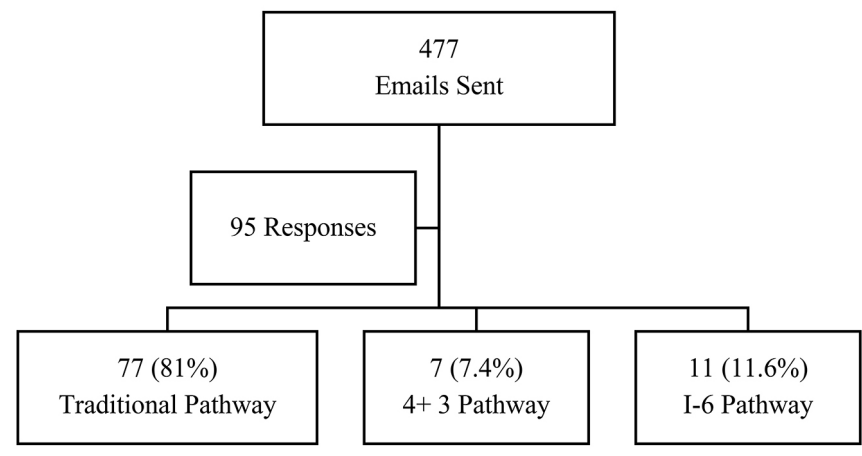

Figure 2. Survey response flowchart for training pathways

pass rate, and overall perceptions of preparation for and satisfaction with a career in CTS. In addition, respondents were given the opportunity to provide comments regarding the advantages and disadvantages of their respective training experience with free-text fields. (Figure 1)

Cardiothoracic surgeons in their first five years of practice, who graduated from CTS residency from 2012-2017, were eligible to take the survey. This range was selected because it included the time period during which all three training 
Table 1. Specialized Training During Residency (Data listed as N (\%)

\begin{tabular}{lcccc}
\hline Formal Training During Cardiothoracic Residency & Yes $(\%)$ & Traditional Pathway $(N=77)$ & 4+3 Pathway $(N=7)$ & I-6 Pathway $(N=11)$ \\
\hline Echocardiography & $22(23.4)$ & $14(18.1)$ & $0(0)$ & $0(0)$ \\
Interventional cardiology & $17(18.0)$ & $9(11.7)$ & $32(72.7)$ \\
Interventional pulmonology & $37(39.4)$ & $31(40.3)$ & $3(0)$ & $5(42.7)$ \\
Endovascular & $43(45.7)$ & $49(63.6)$ & $6(85.7)$ & $6(85.7)$ \\
Minimally invasive cardiothoracic surgery & $63(67.0)$ & $34(44.2)$ & $5(71.4)$ & $9(81.8)$ \\
Robotic surgery & $44(46.8)$ & $68(88.3)$ & $6(85.7)$ & $9(36.7)$
\end{tabular}

paradigms existed. An email list was generated and a link to the online survey was sent to those surgeons, who met the inclusion criteria, with two reminder emails sent three weeks apart to non-responders. The survey was closed after twomonths. The primary endpoints were adequate career preparation and career satisfaction, which were scored on a rating scale from 1-100, 100 being the most positive. All study data were collected anonymously and managed using REDCap (Research Electronic Data Capture), an electronic data capture tool. Responses were computed and presented as frequencies. Free-text responses were qualitatively interpreted. An analysis of variance (ANOVA) test was performed for the primary endpoints of the study. A two-sided $P$-value less than 0.05 was predetermined to be statistically significant.

\section{RESULTS}

Demographics: Four hundred seventy-seven emails were sent. Ninety-five cardiothoracic surgeons completed the survey, for a response rate of $20 \%$. Seventy-six of the 95 respondents (80\%) were male with a mean age of 39 years old (range 33-50). Of the 95 survey participants, $77(81.0 \%)$ completed a traditional training pathway, 7 (7.4\%) completed a $4+3$ pathway, and $11(11.6 \%)$ completed an I-6 pathway. (Figure 2) The average number of graduating fellows was 2.2 per year (range 1-10).

Training pathway and residency satisfaction: Seventyseven out of the 95 respondents completed a traditional pathway. Seventy-six $(98.7 \%)$ completed the entire survey. Of these, $71(93.4 \%)$ felt prepared for fellowship at the completion of their general surgery residency.

Seven respondents completed a $4+3$ training pathway and all of them completed the entire survey. All surgeons who completed the $4+3$ pathway started cardiothoracic training during their 4th year of residency. Overall, respondents felt that their general surgery rotations gave them a good foundation of medical knowledge, patient care, and technical skills. Six $(85.7 \%)$ felt they were included as members of the team on their general surgery rotations.

Eleven respondents completed an I-6 training pathway, and 10 of them completed the entire survey. Eight (72.2\%) felt they were considered to be part of the CTS department in their intern year, and all felt that they were treated as members of the team on their non-cardiothoracic surgery rotations. Overall, respondents felt that their non-cardiac surgery rotations were helpful, especially in learning wire skills, echocardiography, and cardiac imaging.

Ninety-four out of the 95 respondents (99\%) completed survey questions, regarding formal specialized training during their training. Twenty-two $(23.4 \%)$ had formal echo training, $17(18.0 \%)$ had formal interventional cardiology training, $43(45.7 \%)$ had formal endovascular training, $63(67 \%)$ had formal minimally invasive CTS training, and 37 (39.4\%) had interventional pulmonology training. Table 1 summarizes the formal specialized training completed by the survey respondents and the degree to which that training is incorporated into their current surgical practice. (Table 1)

Twenty-six out of the 95 surgeons $(27 \%)$ surveyed completed additional training beyond CTS residency-11 (42.3\%) transplant/mechanical circulatory support, 5 (19.2\%) additional thoracic, 4 (15.4\%) aortic/endovascular, 3 (11.5\%) congenital, $2(7.7 \%)$ robotics, and $1(3.8 \%)$ additional cardiac. Of the 26 respondents who completed an additional fellowship, $19(73.0 \%)$ completed a traditional training pathway, $1(3.8 \%)$ completed a $4+3$ pathway, and $2(7.7 \%)$ completed an I-6 pathway. (Figure 3) Twenty-three (24.0\%) completed $\geq 10$ years of post-graduate training. In addition, $49(51.6 \%)$ reported participating in academic enrichment during residency. Of these 49 surgeons, $11(22.4 \%)$ obtained a Master's degree, and 38 $(77.5 \%)$ participated in research with 15 out of these 38 participants (39.5\%) reporting 1 to 3 years of dedicated research time.

Among all survey participants, most felt adequately prepared for practice with a mean response of 79.8 (range 31-100). Within each training program, the mean response for adequate training and preparedness was 78.5 (range 31-100), 75.9 (range 52-95), and 92.5 (range 68-100) for traditional, $4+3$, and I-6 pathways, respectively $(P=0.05)$.

Board Examination Pass Rate - Written Board Examination: Of the 95 respondents, 88 (92.6\%) took the written boards within the first year of finishing training, and 87 out of the $88(98.9 \%)$ passed. The remaining 7 graduates took the written boards within the first 2 years of graduation and all 7 (100\%) passed - 6 of these graduates completed a traditional training pathway, and 1 graduate completed a $4+3$ pathway. 

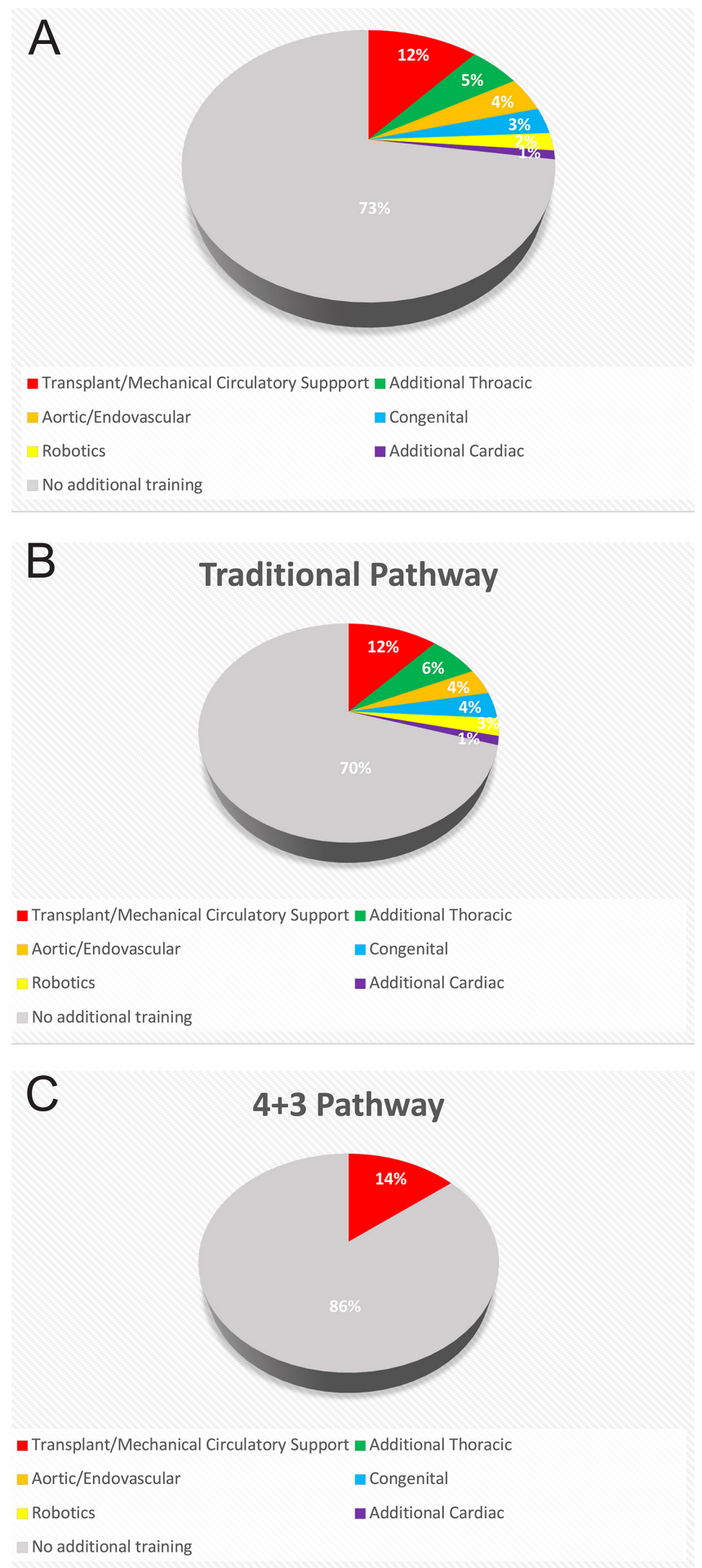

Figure 3. Advanced training

a. Types of Advanced Training Completed

b. Types of Advanced Training Completed by Traditional Pathway Graduates

c. Types of Advanced Training Completed by 4+3 Pathway Graduates

d. Types of Advanced Training Completed by I-6 Pathway Graduates
Oral Board Examination: Of the 95 respondents, 84 $(88.4 \%)$ took the oral boards within the first year of finishing training, $10(10.5 \%)$ within 3 years, and 1 graduate did not respond. The first-year pass rate was $91.7 \%(77 / 84)$. The mean number of attempts to pass both the written and oral boards was 1.22 (range 1-5), with 82 out of 95 graduates (86.3\%) passing both on the first attempt. Eighty-six out of the $95(90.5 \%)$ survey participants indicated that they believed their surgical training adequately prepared them to take the thoracic board examination in their 1st year after training. One graduate $(1 \%)$ from a traditional pathway did not believe that his training program provided adequate board preparation, and 8 graduates $(8.4 \%)$ did not respond.

Practice Type and Career Satisfaction: When asked to define his/her current specialty, 36 of the 95 respondents $(37.9 \%)$ classified themselves as primarily cardiac, $2(2.1 \%)$ cardiac/other, $31(32.6 \%)$ thoracic, $1(1.0 \%)$ thoracic/other, $20(21.0 \%)$ cardiac/thoracic, $2(2.1 \%)$ congenital, and 3 $(3.2 \%)$ a combination of 3 of the specialties. (Table 2) Subspecialties varied widely and included robotics, arrhythmia surgery, transplant/mechanical circulatory support, congenital heart transplant, structural heart, endovascular, minimally invasive, aortic/mitral valve, aortic, general thoracic and vascular, thoracic oncology, and esophageal. In terms of practice type, $51(53.7 \%)$ identified their practice as academic, 23 $(24.2 \%)$ private practice, $8(8.4 \%)$ hospital employed private practice, $9(9.5 \%)$ private practice with academic affiliation or research, $3(3.2 \%)$ military, and $1(1.0 \%)$ locum tenens.

Overall, mean career satisfaction was 87.6 (range 37-100), with a mean of 86.0 (range 37-100), 95.4 (range 80-100), and 95.8 (range $84-100$ ) for traditional, $4+3$, and I-6 pathways, respectively $(P=0.08)$.

Perceived Advantages and Disadvantages of Individual Training Programs: Participants were asked to identify the 3 main deficiencies across their programs, and 85 out of 95 participants responded. The most common responses included lack of operative independence/autonomy $(13 / 85,15.3 \%)$, poor or non-existent didactics $(11 / 85,12.9 \%)$, lack of research opportunities $(7 / 85,8.2 \%)$, and poor teaching from attendings or excessive politics with attendings $(7 / 85,8.2 \%)$. While work hour violations were a concern expressed by several participants (5/85, $5.9 \%$ ), the main critique was focused on the lack of support staff (i.e. co-residents, advanced practice providers, etc). Perceived surgical deficiencies included experience with robotic training $(12 / 85,14.1 \%)$, heart and lung transplant $(10 / 85,11.8 \%)$, minimally invasive cardiac surgery $(8 / 85,9.4 \%)$, mitral valve surgery $(7 / 85,8.2 \%)$, transcatheter aortic valve replacement $(6 / 85,7.0 \%)$, and lack of esophageal training (benign, malignant, minimally invasive) $(6 / 85,7.0 \%)$. Absence of a formalized mechanical circulatory support training $(8 / 85,9.4 \%)$, in addition to lack of formal training outside of cardiothoracic surgery including areas such as endovascular and wire skills (14/85, $16.4 \%)$, echocardiography $(7 / 85,8.2 \%)$, cardiac catheterization $(7 / 85,8.2 \%)$, and interventional pulmonology $(5 / 85,5.9 \%)$ also were common comments among survey participants.

When surveyed about which pathway respondents would choose in the current era of CTS education and training, 52 out of 95 respondents $(54.7 \%$ ) would choose a traditional pathway, 
Table 2. Current Specialty Breakdown (Data listed as N (\%)

\begin{tabular}{lcccc}
\hline Specialty & Overall (\%) & Traditional Pathway (\%) & 4+3 Pathway (\%) & $1-6$ Pathway (\%) \\
\hline Cardiac & $36(37.9)$ & $23(30.0)$ & $4(57.1)$ & $9(81.8)$ \\
Cardiac/Other & $2(2.1)$ & $1(1.3)$ & $1(14.3)$ & $0(0)$ \\
Thoracic & $31(32.6)$ & $31(40.3)$ & $0(0)$ & $0(0)$ \\
Thoracic/Other & $1(1.0)$ & $1(1.3)$ & $2(28.6)$ & $0(0)$ \\
Cardiac/Thoracic & $20(21.0)$ & $16(20.8)$ & $0(0)$ & $0(0)$ \\
Congenital & $2(2.1)$ & $2(2.6)$ & $0(0)$ & $0(0)$
\end{tabular}

$17(17.9 \%)$ a $4+3$ pathway, and $19(20.0 \%)$ an I-6 program; 7 $(7.4 \%)$ did not respond. Of the 52 who would choose a traditional pathway, all had completed a traditional pathway. These respondents believed that their general surgery training provided a good foundation on which to build upon during fellowship and that general surgery skills helped with general thoracic surgery. Of the 17 respondents who selected the $4+3$ pathway, $14(82.3 \%)$ had originally completed a traditional pathway, and $3(17.6 \%)$ had originally completed a $4+3$ pathway. The $4+3$ pathway was attractive to these respondents, due to the shorter training time while still receiving a broad and comprehensive surgical education. Of the 19 respondents who selected the I-6 pathway, $6(31.6 \%)$ had completed a traditional pathway, 3 (15.8\%) completed a 4+3 pathway, and 10 (52.6\%) had originally completed an I-6 pathway. In fact, 10/11 of the I-6 trainees would choose an I-6 program over other training pathways; $1 / 11$ did not answer that survey question. The reason for choosing an I-6 program was more focused training with shorter overall training time. Interestingly, 20 of the 72 (27.8\%) traditional pathway trained surgeons chose one of the integrated pathways; of those 20 surgeons, $55 \%$ practice cardiac surgery, $20 \%$ cardiac and thoracic surgery, and $25 \%$ thoracic surgery. Additionally, $70 \%$ of them did not complete additional training suggesting that the length of training played a role in their decision.

\section{DISCUSSION}

This study is unique from previous publications as it directly asks one to address their perceptions of training, based on their individual experience and current practice. To our knowledge, this is the first survey aimed at assessing how recent cardiothoracic surgery graduates up to 5 years in practice perceived their training with representative proportions from all 3 training pathways. Based off of the current number of CTS training programs in the United States, $63.2 \%$ of programs are traditional, $11.1 \%$ are $4+3$, and $25 \%$ are I-6; in this study the respondents were $81 \%$ traditional, $7.4 \% 4+3$, and 11.6 I-6making this survey fairly representative of the current mix of trainees. Overall, at the completion of their CTS training, surgeons felt well prepared for practice and were satisfied with their career choice. Data collected from this survey suggests that regardless of the training program selected, early career surgeons were adequately prepared by their respective training programs, as reflected by their overall feedback from the survey questions as well as objective data from their written and oral board pass rate. However, despite these overall sentiments, the biggest concerns identified from this survey respondents were didactic teaching and exposure to surgical and non-surgical subspecialties, such as echocardiography, robotic surgery, and mechanical circulatory support. These findings are similar to those reported in Chu et al.'s survey from the 2014 ABTS oral examination [Chu 2016]. The concerns of these surgeons parallel the evolving field of cardiothoracic surgery and the need for an ever-expanding skill set and ability to subspecialize.

The question of which training pathway provides the optimal training and which is the most appealing is not as clear as one might expect. Interestingly, in this survey, when asked which training pathway participants would choose if given a second chance, all surgeons who trained in an integrated thoracic surgery residency, either the $4+3$ or I- 6 track, would choose an integrated pathway again. Whereas, those who completed a traditional pathway were more divided. Over $25 \%$ would choose either the $4+3$ or I- 6 track primarily to reduce the number of years of training. Lebastchi et al. sent a survey to I-6 program directors aimed at assessing current trainees. Most I-6 directors believed their residents were more skilled, had more academic interest, equivalent maturity, were better trained, better prepared for advances in the field of CTS, and had superior comprehension of CTS disease processes compared with their traditionally trained colleagues. Furthermore, the majority of I-6 program directors believed that the I-6 format represents a natural evolution toward improved residency training, and $67 \%$ would have chosen an I-6 program for their own training [Lebastchi 2014]. Similarly in a survey of I-6 residents, $95 \%$ were satisfied with their program [Lebastchi 2014]. Our survey found that 100\% of I-6 trained cardiothoracic surgeons would choose an I-6 pathway again.

The need or desire for additional training after CTS residency also is of interest. Over a quarter of respondents completed additional training; when analyzed by pathway, those who trained under a traditional pathway had a 30\% rate of completing additional training as compared with $14 \%$ for a $4+3$ pathway and $9 \%$ for an I-6 pathway. Other reported rates of additional training vary across studies-in the 2010 Thoracic Surgery Residents Association (TSRA) survey, 39\% of respondents planned 
on additional fellowship training [Sarkaria 2011]. In a survey of just I-6 residents, $88 \%$ anticipated additional training beyond residency [Lebastchi 2014]. In data taken from the TSRA inservice training exam survey from 2003-2014, interest in additional training increased from $10 \%$ in 2003 to $47 \%$ in 2014 [Stephens 2015]. It is important to note that all of these surveys were taken while residents were still in training and rates could differ when training concluded as opposed to our study, where the participants already had completed all of their training. Chu et al.'s survey of recent graduates, found that $42 \%$ completed additional training, keeping in mind that $95 \%$ of participants in that study had completed a traditional pathway [Chu 2016].

Twenty percent of our survey participants were female; other surveys have similar findings with a range from $17.4 \%$ to $24 \%$, although these were trainees as opposed to practicing surgeons [Lebastchi 2014; Stephens 2016; Chu 2016]. Although women comprise approximately $5 \%$ of practicing cardiothoracic surgeons, $20 \%$ of current cardiothoracic surgery residents are women, and our study's demographics likely represent this evolving increase of female CTS graduates [Stephens 2016].

There are several limitations to this study. The first major limitation of any survey study is the list of recipients. While we compiled a comprehensive list of email addresses, due to changes in email addresses from training onward, there were some surgeons who met the study criteria but were unable to be reached via email. The response rate of $20 \%$, while not atypical for email surveys, could limit the generalization of our findings. While the percentage breakdown of participants by training pathway mirrors the national breakdown, those who responded had a higher board pass rate $(99 \%$ vs. $81-95 \%$ 2014-2018 written boards and $92 \%$ vs. $72-84 \%$ 2015-2019 oral boards [American Board of Thoracic Surgery]), suggesting a selection bias of a more productive training experience which could affect the opinions on participants' training. Additionally, despite free text response opportunities in the survey, we may not have fully captured the reasoning for why participants would choose a different training pathway-for example, educational debt, gender and racial disparities, which have surfaced in other surveys [Lebastchi 2014; Lee 2003; Sarkaria 2011, Stephens 2016; Chu 2016]. Finally, over $80 \%$ of the respondents completed a traditional training pathway, which ultimately could bias the results to reflect the opinions of those surgeons. Currently, with the limited number of $4+3$ and I- 6 graduates, the distribution of training pathway respondents is likely a representative survey, however in the coming years as the number of integrated graduates increase, future responses to these same questions may shift to reflect changes in these evolving training pathways. Nevertheless, these data are hypothesis generating and can guide further prospective studies into answering some of these questions more definitively.

To our knowledge, this is the first survey to collect data addressing perceptions of training based on individual experience and current practice from a representative population of early career cardiothoracic surgeons from all 3 training pathways. Overall, participants trained across all three pathways felt well prepared and satisfied with their career. Yet over 25\% of those who completed a traditional pathway would choose an integrated pathway of either a $4+3$ or I-6, if given the option.
In comparison, $100 \%$ of $4+3$ and I- 6 pathway trained surgeons would choose an integrated pathway again. Although no one training pathway was preferred to another, data from this study provides insights to help the specialty better understand how to continue improving cardiothoracic surgical training to prepare the next generation of cardiothoracic surgeons to meet the challenges of an ever-evolving field.

\section{ACKNOWLEDGEMENT}

The funding for this study was provided by the Eastern Cardiothoracic Surgical Society.

\section{REFERENCES}

Alexander J. 1936. The Training of a Surgeon who Expects to Specialize in Thoracic Surgery. J Thorac Surg. (5): p. 579-582.

American Board of Thoracic Surgery. 5-Year Pass Rate. [cited 2020 July 10]; Available from: https://www.abts.org/ABTS/CertificationWebPages/Examination_Sequence.aspx.

Chu D, et al. 2016. Are There Gaps in Current Thoracic Surgery Residency Training Programs? Ann Thorac Surg. 101(6): p. 2350-5.

Crawford FA, Jr. 2005. Thoracic surgery education--past, present, and future. Ann Thorac Surg, 79(6): p. S2232-7.

Ikonomidis JS, Crawford FA, Jr., Fann JI. 2014. Integrated surgical residency initiative: implications for cardiothoracic surgery. Semin Thorac Cardiovasc Surg. 26(1): p. 14-23.

Lebastchi AH, et al. 2014. First nationwide survey of US integrated 6-year cardiothoracic surgical residency program directors. J Thorac Cardiovasc Surg. 148(2): p. 408-15 e1.

Lebastchi AH and Yuh DD. 2014. Nationwide survey of US integrated 6-year cardiothoracic surgical residents. J Thorac Cardiovasc Surg. 148(2): p. 401-7.

Lee R. 2003. Help wanted. Ann Thorac Surg. 76(6): p. 1779-81.

Nguyen TC, et al. 2015. Resident Perceptions of 2-Year Versus 3-Year Cardiothoracic Training Programs. Ann Thorac Surg. 99(6): p. 2070-5; discussion 2075-6.

Sarkaria IS, et al. 2011. The 2010 Thoracic Surgery Residents Association workforce survey report: a view from the trenches. Ann Thorac Surg. 92(6): p. 2062-70; discussion 2070-1.

Stephens EH, et al. 2015. A Decade of Change: Training and Career Paths of Cardiothoracic Surgery Residents 2003 to 2014. Ann Thorac Surg. 100(4): p. 1305-13; discussion 1313-4.

Stephens EH, et al. 2016. Gender and Cardiothoracic Surgery Training: Specialty Interests, Satisfaction, and Career Pathways. Ann Thorac Surg. 102(1): p. 200-6.

Tchantchaleishvili V, et al. 2015. Current integrated cardiothoracic surgery residents: a Thoracic Surgery Residents Association survey. Ann Thorac Surg. 99(3): p. 1040-7.

Thoracic Surgery Directors Association. 2012. CT Surgery Training Pathways.

Zhu Y, Goldstone AB, Woo YJ. 2019. Integrated Thoracic Surgery Residency: Current Status and Future Evolution. Semin Thorac Cardiovasc Surg. 31(3): p. 345-349. 